\title{
Philosophiques
}

\section{Déontologisme et droits}

\section{Sarah Stroud}

Volume 26, numéro 1, printemps 1999

URI : https://id.erudit.org/iderudit/004988ar

DOI : https://doi.org/10.7202/004988ar

Aller au sommaire du numéro

\section{Éditeur(s)}

Société de philosophie du Québec

\section{ISSN}

0316-2923 (imprimé)

1492-1391 (numérique)

Découvrir la revue

\section{Citer cet article}

Stroud, S. (1999). Déontologisme et droits. Philosophiques, 26(1), 139-148.

https://doi.org/10.7202/004988ar

\section{Résumé de l'article}

Dans ce texte, l'accent est mis sur les contraintes ou restrictions dites déontologiques. Croire en l'existence de telles contraintes, c'est croire qu'il peut être moralement inadmissible de faire quelque chose, même si cette action se révélait la seule manière d'empêcher un résultat encore pire. La question que pose et examine ce texte est celle de savoir pourquoi il est mal de faire des actions qui semblent violer une contrainte déontologique. Plus particulièrement, ce texte étudie l'hypothèse séduisante que nous pourrions expliquer ce qu'il y a de mal dans ce type d'actions en termes de droits. L'article argumente que cette idée, bien que toute naturelle, ne nous permettra pas d'expliquer de manière satisfaisante nos convictions déontologiques. Les droits peuvent être interprétés de plusieurs manières différentes, mais peu importe l'option choisie, nous rencontrons des difficultés. Le problème principal est que l'appel aux droits fait souvent usage des jugements d'admissibilité que nous essayons d'expliquer. Le supposé explanans n'a donc pas l'indépendance requise face à l' explanandum pour en constituer une véritable explication. 


\title{
Déontologisme et droits
}

\author{
SARAH STROUD \\ sarah@philo.mcgill.ca \\ Université McGill \\ Traduit de l'anglais par France Émond et l'auteur
}

\begin{abstract}
RÉSUMÉ. - Dans ce texte, l'accent est mis sur les contraintes ou restrictions dites déontologiques. Croire en l'existence de telles contraintes, c'est croire qu'il peut être moralement inadmissible de faire quelque chose, même si cette action se révélait la seule manière d'empêcher un résultat encore pire. La question que pose et examine ce texte est celle de savoir pourquoi il est mal de faire des actions qui semblent violer une contrainte déontologique. Plus particulièrement, ce texte étudie l'hypothèse séduisante que nous pourrions expliquer ce qu'il y a de mal dans ce type d'actions en termes de droits. L'article argumente que cette idée, bien que toute naturelle, ne nous permettra pas d'expliquer de manière satisfaisante nos convictions déontologiques. Les droits peuvent être interprétés de plusieurs manières différentes, mais peu importe l'option choisie, nous rencontrons des difficultés. Le problème principal est que l'appel aux droits fait souvent usage des jugements d'admissibilité que nous essayons d'expliquer. Le supposé explanans n'a donc pas l'indépendance requise face à l'explanandum pour en constituer une véritable explication.
\end{abstract}

ABSTRACT. - The focus of this paper is deontological restrictions or constraints. To believe in the existence of such constraints is to think that it can be morally impermissible to do something even if doing that is the only way to prevent an even worse outcome. The question which this article poses and considers is why it is wrong to perform acts which seem to violate a deontological restriction. In particular, the paper investigates the attractive possibility that we could explain this wrongness in terms of rights. I argue that this idea, while natural, will not yield a satisfying explanation of our deontological convictions. Rights can be understood in several different ways, but no matter which option we take we encounter difficulties. The principal problem is that appeals to rights often make use of the very judgments of permissibility which we are trying to explain. The supposed explanans thus lacks sufficient independence from the explanandum to constitute a genuine explanation of it.

Dans ce texte, l'accent sera mis sur les restrictions ou contraintes dites déontologiques'. Plus particulièrement, notre sujet sera la relation entre ces restrictions et les droits : le texte soulèvera quelques difficultés à propos de l'idée séduisante selon laquelle nous pourrions expliquer les premières en faisant appel aux seconds. Mais tout d'abord, afin de clarifier notre objectif, commençons avec un exemple dont le but est d'illustrer les contraintes déontologiques. Une organisation terroriste planifie une attaque à la bombe qui

I. Dans ce texte, les termes « restrictions " et " contraintes » sont utilisés de manière interchangeable. 
blessera et tuera plusieurs personnes. Vous êtes un agent secret qui a infiltré l'organisation afin d'obtenir plus de renseignements sur cette dernière. Vous êtes encore en marge du groupe et, de ce fait, vous n'avez pas encore assez de renseignements sur la cellule pour empêcher l'attaque de se produire. (Vous savez également que vos collègues au quartier général n'ont pas ces renseignements non plus.) Cependant, vous en connaissez assez sur l'organisation pour savoir qui sont les terroristes suffisamment haut placés dans la chaîne de commandement pour obtenir les renseignements dont vous avez besoin. Supposons qu'il n'y ait aucune autre manière d'empêcher l'attaque à l'explosif que de torturer devant son père l'enfant d'un des terroristes pour que ce dernier vous révèle les renseignements. Dans ce cas, devriez-vous torturer l'enfant? La plupart d'entre nous répondraient par la négative, que vous ne devez pas même envisager une telle option, tout en admettant que l'éventuelle bombe terroriste serait beaucoup plus nocive en termes de résultats ${ }^{2}$.

Des jugements moraux comme celui-ci semblent exprimer une croyance en l'existence de restrictions déontologiques sur la conduite. Croire en l'existence de contraintes déontologiques, c'est croire qu'il peut être moralement inadmissible de faire quelque chose même si cette action se révélait la seule manière d'empêcher un résultat encore pire ${ }^{3}$. À propos du présent exemple, plusieurs d'entre nous pensent $e t$ qu'il serait mal de torturer l'enfant, et que l'état des choses dans lequel l'explosion se produit serait pire que le scénario où l'enfant est torturé. (Dans le premier cas, après tout, un plus grand nombre de personnes seraient plus sérieusement lésées que dans le second.) Notre verdict ici - s'il renvoie en effet à une croyance en des contraintes marque ainsi un contraste net entre la conception morale de la plupart d'entre nous et toute conception conséquentialiste qui exige que l'agent, dans une situation donnée, choisisse l'action à faire en fonction des meilleurs (ou des moins pires) résultats possibles ${ }^{4}$. Les théories conséquentialistes considèrent la

2. Ce cas est une version modifiée d'un exemple présenté par Samuel Scheffler (voir Scheffler 1988, p. 3).

3. Il faut admettre que ce ne sont pas tous les déontologistes qui offriraient une telle caractérisation des contraintes ou des restrictions. Car certains d'entre eux (voir Thomson 1997 et Foot 1985) estiment que l'idée d'un état des choses meilleur ou pire n'a pas de signification claire. Quelqu'un qui soutient cette position n'emploierait certainement pas l'idée d'un résultat encore pire comme je le fais dans ce texte (et, paraît-il, comme la plupart d'entre nous le feraient).

4. La qualification "s'il renvoie en effet à une croyance en des contraintes " est nécessaire pour la raison suivante. Dans tout cas particulier, le conséquentialiste peut argumenter qu'il peut en arriver à la conclusion qu'il serait mal de faire $\mathrm{X}$ sans en appeler aux restrictions déontologiques. C'est-à-dire qu'il peut contester, pour tout exemple spécifique que ce soit, qu'il s'agisse véritablement d'un exemple pour lequel il est moralement défendu de faire $\mathrm{X}$, même si faire $\mathrm{X}$ produisait un résultat moins dommageable dans l'ensemble. Une croyance selon laquelle il serait mal de torturer l'enfant (par exemple) n'est donc pas une preuve concluante de la croyance en des contraintes déontologiques. Seule une personne qui entérine la caractérisation générale des contraintes présentée plus haut dans le texte pourra nécessairement être considérée comme croyant en de telles contraintes. 
comparaison des conséquences d'un point de vue impersonnel comme étant la seule base de l'évaluation morale des actions. Mais, comme nous l'avons vu, il paraît impossible que ce qu'il y ait de mal dans le fait de torturer l'enfant vienne d'une telle appréciation comparative des conséquences, laquelle favoriserait (selon toute apparence) la torture de l'enfant.

Il vaudrait la peine d'essayer de mieux comprendre ces convictions déontologiques. Pourquoi est-il mal de torturer l'enfant du terroriste afin d'obtenir les renseignements nécessaires pour empêcher l'attaque du groupe? À quoi répondons-nous exactement, en jugeant qu'une telle action est mal dans de telles circonstances? Quelle peut être la base de cet interdit moral qui persiste même si sa violation amenait des conséquences moins mauvaises dans l'ensemble? Nous gagnerions en clarté vis-à-vis notre conscience morale si nous étions capables de répondre à ces questions interprétatives, si nous comprenions, par exemple, ce qui fait que c'est mal de tuer, même quand un tel acte est un moyen nécessaire pour réduire le nombre total de morts. Cet article poursuit ce projet explicatif, même s'il ne propose que le rejet d'une réponse naturelle. Il est tentant de supposer que nous pouvons en appeler aux droits pour expliquer le mal que les restrictions déontologiques expriment. Mais ce texte offrira des arguments pour montrer qu'un tel recours ne constituera pas une explication satisfaisante.

L'étude proposée ici se distingue des discussions typiques du déontologisme dans la littérature récente. De façon surprenante, les défenseurs et les détracteurs des restrictions déontologiques sont souvent d'accord dès le départ sur l'avis que les contraintes déontologiques sont paradoxales prima facie. Étant donné ce point de départ commun, le fardeau de la preuve paraît revenir aux conceptions morales déontologiques : ces théories sont obligées de porter une charge particulièrement lourde non partagée avec les autres conceptions morales. Dans un tel contexte argumentatif, les questions du genre "pourquoi " mentionnées plus haut prennent facilement un ton différent : elles deviennent accusatrices, voire menaçantes. Elles nous rappellent le danger du paradoxe qui est censé entourer l'idée des restrictions déontologiques. La tâche des défenseurs de ces contraintes devient donc lourde. Il sont invités à offrir un raisonnement en faveur des contraintes qui réussit à dissiper ou à annuler le semblant de paradoxe. S'ils ne parviennent pas à faire cela, les opposants concluront que les nombreux jugements qui semblent exprimer notre croyance en de telles contraintes devraient être rejetés comme étant des éléments irrationnels de notre conception morale.

Le projet du présent texte se distingue en son point de départ, et ce, parce que je ne suis pas convaincue par les raisons avancées pour qualifier d'irrationnels (même prima facie) les engagements déontologiques 5 . Je ne trouve ni sage ni nécessaire que le déontologiste accepte un fardeau de la preuve spécialement lourd sur la foi de ces raisons. Cet article suppose donc qu'aucune charge de ce genre ne puisse être déposée avec succès sur les épaules

5. Mon texte non publié « Deontology : Lifting the Burden » explique le pourquoi de cette position. 
du déontologiste. Mais nous aimerions quand même mieux comprendre les fondements de ces jugements. Supposons qu'il n'y ait pas de fardeau de la preuve spécifique qu'il incombe au déontologisme d'écarter afin de se prémunir d'un rejet. Toutefois, il vaudrait la peine d'obtenir une plus grande clarté dans notre conception morale en comprenant mieux la nature du mal que les violations de contraintes semblent incarner. Ce texte constitue donc un exercice d'auto-compréhension ou, si vous préférez, de compréhension morale, pas une opération de sauvetage.

Revenons maintenant à notre question interprétative. En particulier, examinons une réponse naturelle à la question de savoir pourquoi il est mal de torturer l'enfant, ou de faire des choix similaires dans d'autres exemples souvent utilisés dans la littérature en tant qu'illustrations de contraintes déontologiques. Dans un de ces exemples, vous êtes un chirurgien qui a cinq patients nécessitant une transplantation d'organes pour survivre. Vous pouvez les sauver seulement en tuant un patient en santé qui se trouve là pour un examen de routine et en transplantant ses organes aux cinq autres patients. Dans un autre exemple typique, des membres de la Mafia viennent vous voir et vous expliquent (sincèrement) qu'ils vont tuer cinq hommes d'affaires innocents, à moins que vous ne leur fassiez la faveur de tuer un autre homme d'affaires innocent. Dans tous ces cas, il paraît être interdit de commettre l'acte qui aura les meilleures (ou les moins pires) conséquences. La suggestion à considérer est la suivante : cet acte est mal parce qu'il viole les droits des victimes. L'enfant a un droit à ne pas être torturé; le patient a un droit à ne pas être charcuté; l'homme d'affaires (innocent) a un droit à ne pas être tué. Ces droits, dit-on, sont la base du mal dans chacun de ces cas. En bref, ce à quoi nous répondons en jugeant que ces actions sont inadmissibles, c'est à la violation grossière des droits que chaque cas représente ${ }^{6}$. C'est en fait une pensée naturelle; mais nous verrons dans ce qui suit que cela ne sera pas satisfaisant comme réponse à la question interprétative. Quand nous regardons de plus près ce que c'est qu'avoir un droit, des complications émergent rapidement, lesquelles bloquent un simple recours aux droits en tant que réponse à notre question explicative.

$* * *$

6. J'ai présenté tous ces cas comme étant des illustrations d'un conflit entre l'acte qui amènera les meilleures conséquences et une contrainte déontologique qui interdit cet acte. Mais un évaluateur anonyme pour Philosophiques a suggéré que tous ces cas pourraient être vus d'une autre manière : comme des conflits entre les droits. Dans le cas de la bombe terroriste, par exemple, on doit choisir (selon cette interprétation) entre le droit de l'enfant de ne pas être torturé et les droits des futures victimes de la bombe de ne pas être tuées. Mais cette interprétation ne semble pas bien caractériser toutes les situations où les restrictions déontologiques peuvent entrer en jeu. Par exemple, il serait moralement interdit de tuer délibérément une personne même si on pouvait ainsi éviter la mort de plusieurs autres dans un tremblement de terre ou dans un ouragan. Mais les «actions » d'un tremblement de terre ou d'un ouragan ne peuvent violer les droits de personne; il ne s'agit donc pas dans ces cas d'un choix entre les droits. 
Qu'est-ce que c'est qu'avoir un droit? Un exemple nous révélera immédiatement une difficulté qui semble empêcher une réponse facile à cette question. En effet, dans plusieurs exemples présentés par Judith Jarvis Thomson, deux affirmations sur les droits que nous aurions crues incompatibles semblent néanmoins être toutes deux vraies (voir Thomson 1976 et 1990, chap. 3; voir aussi 1986b). Ces deux affirmations sont :

a) $\mathrm{S}$ a un droit que $\mathrm{T}$ ne fasse pas $\mathrm{A}$, et

b) il est admissible pour $\mathrm{T}$ de faire $\mathrm{A}$.

Disons que $\mathrm{T}$ enfreint le droit de $\mathrm{S}$ (que $\mathrm{T}$ commet une infraction à ce droit) si $\mathrm{S}$ a un droit que $\mathrm{T}$ ne fasse pas $\mathrm{A}$, et que malgré tout $\mathrm{T}$ fasse $\mathrm{A}$. L'argument de Thomson est qu'il semble y avoir des situations dans lesquelles il est admissible d'enfreindre un droit.

Supposons par exemple que Georges doive traverser le terrain de Nicole en courant afin d'arriver à l'hôpital à temps pour recevoir un traitement nécessaire à sa survie. Nicole ne lui a pas donné d'autorisation d'être sur sa propriété. En courant sur le terrain de Nicole, Georges s'introduit donc sans permission sur la propriété de Nicole, et ainsi, vraisemblablement, enfreint le droit de propriété de Nicole ${ }^{7}$. Nous sommes donc enclins à dire que a) est vraie dans ce cas. (Disons que $\mathrm{S}=$ Nicole, $\mathrm{T}=\mathrm{Georges}$, et $\mathrm{A}=\mathrm{e}$ tre sur le terrain de Nicole sans son autorisation.) Mais assurément, il est néanmoins moralement admissible pour Georges de courir à travers le terrain de Nicole dans de telles circonstances. Donc b) aussi semble être vraie. Nous aurions pu penser que a) et b) sont en général incompatibles, qu'avoir un droit signifie qu'il est toujours inadmissible pour quelqu'un d'enfreindre ce droit. Sinon, d'où viendrait la force morale spéciale des droits? Mais si a) et b) sont vraiment incompatibles, cet exemple semble prouver que nous n'avons pas les droits de propriété que nous avons cru avoir. En particulier, il semble, paradoxalement, que nous n'avons aucun droit qui empêche les gens de s'introduire sans permission sur notre propriété. C'est une conclusion inconfortable; il vaut mieux alors essayer de tirer une autre conclusion de l'exemple.

Le diagnostic qu'offre Thomson est que a) et b) sont toutes deux vraies dans ce cas. Les droits ne sont pas donc absolus : il n'est pas toujours inadmissible d'enfreindre un droit. Thomson résume sa position ainsi : toute infraction à un droit n'est pas une violation d'un droit (Thomson 1976, p. 40; 1990, p. 122). Si T fait ce que $S$ a un droit que T ne fasse pas, il enfreint le droit de S. Mais c'est seulement s'il le fait de manière inadmissible qu'il viole le droit de S. Appelons ceci la conception non-absolutiste des droits. Nous explorerons d'abord les implications de cette conception non-absolutiste pour l'explication du déontologisme. Ensuite, nous examinerons d'autres réponses que l'on pourrait donner aux exemples comme ceux que présente Thomson.

Maintenant que nous avons en main une distinction entre enfreindre un droit et violer un droit, nous devons choisir celui de ces deux termes que nous

7. Georges n'enfreint pas le droit à la propriété de Nicole, c'est-à-dire le droit de posséder quelque chose. Il enfreint plutôt le droit que détient Nicole de jouir en exclusivité de sa propriété (un aspect important du droit de propriété). Je tiens à remercier un évaluateur anonyme pour Philosophiques qui a offert cette clarification. 
voulons utiliser pour expliquer ce qu'il y a de mal dans une violation d'une restriction déontologique. Supposons que nous choisissions le premier. Toutefois, comme nous venons de le noter, dire qu'un acte enfreint les droits de quelqu'un ne garantit pas qu'il soit inadmissible d'accomplir un tel acte, parce que les infractions à un droit sont parfois admissibles. Qu'un acte enfreigne un droit ne peut donc pas être l'entière explication de ce qui est mal dans l'acte. Il doit y avoir quelque chose au-delà de l'infraction à un droit qui contribue à faire de cet acte quelque chose de mal. C'est à dire qu'un simple appel à l'infraction à un droit ne sera pas suffisant en tant que manifestation de ce qu'il $\mathrm{y}$ a de mal dans un acte.

Nous pourrions alors changer d'avis et dire que les restrictions déontologiques interdisent la violation des droits. Mais maintenant, une nouvelle difficulté s'élève. Nous ne pouvons en appeler au fait putatif qu'un acte violerait les droits de quelqu'un pour expliquer pourquoi cet acte est mal. Car juger qu'un acte viole plutôt qu'il enfreint simplement les droits de quelqu'un, c'est déjà juger que l'acte est inadmissible : précisément le jugement que nous cherchons à expliquer. Il est suffisant pour établir le mal qu'un acte viole véritablement un droit; mais le jugement qu'un acte particulier viole un droit semble être simplement la conjonction de l'affirmation qu'il enfreint un droit avec l'affirmation que cette infraction est inadmissible. Ainsi, nous ne pouvons pas nous tourner vers l'idée des violations des droits pour expliquer ce qui est mal dans la transgression d'une contrainte déontologique.

Alors, nous pourrions revenir sur nos pas et nous demander si nous avions raison d'endosser le diagnostic de Thomson dans les exemples qu'elle a présentés. Peut-être était-ce notre acceptation trop rapide du caractère non-absolu des droits qui a soulevé de telles difficultés pour une explication en termes de droits? De surcroit, nous pourrions nous sentir inconfortables avec la conclusion que les droits ne sont pas absolus et qu'ils peuvent être enfreints de façon admissible. Qu'est-il arrivé à l'idée séduisante que les droits sont des atouts ${ }^{8}$ ? À cette idée qu'ils réduisent au silence, rendent caduque la possibilité d'arguments moraux en faveur d'une conclusion opposée? Nous aimons à penser que les droits sont comme des cartes d'atout, et il est difficile de voir comment cette position peut être maintenue une fois que nous abandonnons la déduction allant de " $\mathrm{S}$ a un droit que $\mathrm{T}$ ne fasse pas A » à « il serait mal pour T de faire $\mathrm{A}$ ».

Il vaut la peine de nous arrêter un moment sur l'examen des implications de cette analogie séduisante selon laquelle les droits sont des atouts. Car il est possible de montrer que même cette idée ne nous amène pas à la conclusion que les droits sont absolus. Tout joueur de bridge connaît le supplice qui se produit quand une de ses cartes d'atout, en principe gagnante, est battue à cause d'un dispositif de priorité dans les atouts, lequel fait ainsi triompher l'ennemi. Même si toute carte d'atout bat toute autre carte qui n'est pas de l'atout, c'est néanmoins une triste réalité du bridge — et, je le suggère, de la vie

8. Sur ce point, voir Dworkin 1977. 
morale - que la reine des atouts bat le trois des atouts. Dire que les droits sont des atouts équivaut à dire que le respect des droits prend toujours la priorité morale sur toute autre considération, telle que l'intérêt général. Une fois qu'un droit entre dans l'équation morale, alors il n'importe plus de savoir la somme de « bien » qui serait générée par une option qui transgresserait ce droit. C'està-dire qu'une carte d'atout bat toute carte qui n'est pas de l'atout, même un as. Mais comme le bridge nous le montre, ceci ne nous permet pas de déduire que toute carte d'atout jouée sera toujours gagnante. En particulier, quand une autre carte d'atout a déjà été jouée, votre atout peut ne pas remporter la mise.

S'il y a des conflits de droits - des situations dans lesquelles, peu importe ce que vous faites, vous enfreindrez un droit - alors nous n'avons aucune garantie qu'il soit toujours inadmissible d'enfreindre un droit. En cas de conflit, il est certain que les droits de quelqu'un seront enfreints; et il est possible que cette infraction soit admissible 9 . Nous pouvons voir cela clairement dans les cas où les droits en jeu ne sont pas d'importance égale. Supposons, par exemple, que Charles découvre avec horreur que le tramway qu'il conduit, devenu hors de contrôle, se dirige vers un piéton. Il peut empêcher la mort du piéton seulement s'il attrape un objet sur un des balcons qui défilent le long de son trajet, et qu'il lance cet objet en avant du tramway. S'il fait ceci, il enfreint le droit de propriété du détenteur de l'objet; mais s'il ne le fait pas, il est sur le point d'enfreindre le droit à la vie, un droit bien plus important. Nous convenons, je crois, que dans un tel cas Charles peut prendre cet objet. Ceci est donc un exemple dans lequel une carte d'atout plus forte bat une carte d'atout plus faible. Alors, même si les droits sont des atouts, ils ne sont pas nécessairement absolus.

Pourtant, nous pourrions toujours préférer nous en tenir au caractère absolu des droits, dans l'espoir qu'une telle conception nous permettra de mieux expliquer le déontologisme en termes de droits. Comment, toutefois, pouvons-nous soutenir le caractère absolu des droits lorsque nous nous référons aux exemples de Thomson? La stratégie la plus prometteuse semble être de " rétrécir » le droit de sorte qu'aucun droit ne soit véritablement enfreint dans de telles circonstances. Contrairement à ce que nous aurions pu penser, Georges n'est pas véritablement en train d'enfreindre le droit de propriété de Nicole en traversant sa propriété en courant pour se rendre à l'hôpital. Il est important pour la plausibilité de cette stratégie que nous ne soyons pas forcés de conclure que nous n'avons aucun droit de propriété ou aucun droit pour contrer la circulation d'autrui sur notre terrain. Le résultat envisagé est plutôt que nous devons énoncer plus soigneusement les droits que nous avons. Nous devons limiter leur portée et leur contenu de telle manière que ce qui semble être des exceptions n'en soient pas. Une fois qualifiés de manière appropriée, les droits redeviendront absolus : il sera toujours inadmissible de les enfreindre. Thomson appelle cette stratégie la

9. Je ne prétends pas qu'à toutes les fois qu'il y a un conflit de droits, il y a une option admissible, c'est à dire un droit qui peut être enfreint. Je suggère seulement que cela est à tout le moins vrai quelquefois. 
«spécification » des droits, et nous devrions maintenant examiner si elle est prometteuse dans le contexte de notre démarche explicative.

Thomson suggère qu'il y a deux avenues possibles pour la spécification des droits : la spécification morale et la spécification factuelle (voir Thomson 1976, $\$ 3)$. Selon la première approche, on incorpore dans le droit une qualification portant sur les caractéristiques morales qu'un acte doit contenir afin de constituer une infraction à ce droit. Prenons comme exemple le supposé droit à la vie, que, pourrait-on penser, tout le monde détient. Ce droit ne doit pas être compris comme un droit à ne pas être tué, parce qu'il y a des circonstances dans lesquelles il pourrait être admissible d'enfreindre un tel droit. Plus exactement, nous devrions comprendre le droit à la vie comme (à titre d'exemple) un droit à ne pas être tué injustement. Certainement, il ne serait jamais admissible d'enfreindre ce droit. Il ne pourrait y avoir aucune situation dans laquelle il soit admissible de tuer quelqu'un injustement; la description du meurtre comme étant injuste semble éliminer la possibilité qu'il puisse être moralement admissible. Si nous suivons cette voie, alors nous pouvons maintenir l'idée que nos droits, lorsqu'ils sont bien compris, sont en effet absolus.

La spécification factuelle cherche à obtenir le même résultat, mais en procédant différemment. Plutôt que d'ajouter une qualification précisant une caractéristique morale qu'un acte doit avoir afin de constituer une infraction à un droit, la spécification factuelle tente d'identifier de manière factuelle, c'est-à-dire sans recours à la morale, les circonstances dans lesquelles une infraction apparente n'est pas véritablement une infraction à ce droit. Selon cette conception, le droit à la vie, encore une fois, ne pourrait pas être compris simplement comme un droit à ne pas être tué. Il faut plutôt le voir comme un droit à ne pas être tué sauf s'il s'agit d'une punition pour un crime, ou d'une guerre, ou lors d'un assaut sur autrui, et ainsi de suite. Ces qualifications sont censées s'énoncer seulement en termes de faits non moraux. Comme il semble y avoir un grand nombre de situations différentes dans lesquelles il pourrait être admissible de tuer, les droits incorporeront vraisemblablement beaucoup de qualifications selon cette approche. Mais une fois qualifiés de manière appropriée, les droits seront encore une fois absolus.

Évaluons maintenant ces approches dans le contexte de notre enquête interprétative. Comprendre les droits selon la stratégie de la spécification morale ne nous aidera pas à expliquer pourquoi il est mal de transgresser une restriction déontologique. Si nous pouvons établir qu'une transgression particulière d'une contrainte est vraiment une infraction à un droit absolu, spécifié moralement, cela suffira pour que cet acte soit mal du point de vue moral. Mais serait-ce une explication satisfaisante de la raison pour laquelle un certain acte est mal, de dire qu'il enfreint le droit d'une personne à ne pas être tuée injustement? Cela ne serait pas plus satisfaisant que de dire (comme nous l'avons fait plus tôt) que l'acte est mal parce qu'il constitue une violation et non pas uniquement une infraction à un droit. La difficulté est que le jugement qu'un acte est véritablement une infraction à un droit spécifié 
moralement semble déjà contenir le jugement que l'acte en question n'est pas admissible. Mais cela est exactement le jugement que nous essayons de comprendre et d'expliquer. Nous ne semblons pas faire de progrès sur ce point quand nous tentons d'expliquer notre jugement moral en faisant appel à une infraction à un droit spécifié moralement.

L'approche de la spécification factuelle peut paraître plus prometteuse, parce que la portée d'un droit spécifié de manière factuelle n'est pas limitée par une clause morale explicite équivalente à un jugement d'inadmissibilité. Si nous essayons d'expliquer ce qui est mal dans un acte en disant que c'est une infraction à un droit spécifié de manière factuelle, nous ne nous plaçons pas dans ce cercle très hermétique dans lequel nous nous trouvions avec la spécification morale des droits. Cependant, nous serons quand même enfermés dans un cercle. Si nous nous demandons si un certain acte enfreint véritablement un droit, nous devons nous demander si la spécification factuelle appropriée de ce droit comprendrait une qualification pour le type de situation en question. Afin de déterminer, par exemple, si Georges enfreint le droit de propriété de Nicole, nous devons réfléchir à la spécification factuelle appropriée de ce droit. En particulier, nous devons nous demander si notre droit concernant le passage d'autrui sur notre terrain contient une qualification se référant aux situations dans lesquelles une personne a besoin de se rendre à l'hôpital (ou toute autre catégorie de situations, incluant le présent cas). Sur quelle base allons-nous répondre à cette question?

Rappelons-nous que la démarche vers une spécification détaillée des droits était motivée par le désir de redonner aux droits leur caractère absolu. Ainsi, une spécification particulière de la portée d'un droit en constituera une adéquate seulement si elle mène en effet à un droit absolu, c'est-à-dire à un droit qu'il sera toujours mal d'enfreindre. La question que nous devons nous poser, donc, est de savoir si une exception pour ce type de situation est requise afin de mener à un droit qu'il sera toujours mal d'enfreindre. Mais cela veut dire que nous nous demandons en effet si cet acte est admissible. S'il l'est, nous savons qu'il faut inclure une clause d'exception pour ces types de cas dans la spécification factuelle du droit. S'il ne l'est pas, nous pouvons dire que cet acte enfreint véritablement le droit en question, parce que ce droit (une fois spécifié de manière factuelle) ne contient aucune qualification relative à ce type de cas. Cependant, l'élément clé du jugement que l'acte enfreindrait un droit renvoie encore une fois à l'idée qu'un tel acte ne serait pas admissible. Le problème est que nous décidons en effet de la spécification factuelle appropriée d'un droit en vue de nos jugements sur l'admissibilité. Nous ne pouvons pas, donc, expliquer les seconds en faisant appel à la première.

Révisons les résultats obtenus jusqu'à maintenant dans cet examen. Nous avons commencé avec l'espoir que nous pourrions expliquer ce qu'il y a de mal dans (par exemple) la torture de l'enfant en faisant appel aux droits. Cependant, nous avons dû bien vite faire face à des questions difficiles. Après 
avoir examiné des exemples comme ceux que Thomson présente, voulonsnous adopter une conception absolutiste ou non-absolutiste des droits? C'està-dire, souhaitons-nous dire qu'il est toujours mal d'enfreindre un droit? Si nous suivons la voie suggérée par Thomson et que nous optons pour une conception non-absolutiste des droits, alors nous devons distinguer entre l'infraction et la violation d'un droit. La première n'est pas suffisante pour qu'un acte soit mal, et ainsi, ne peut pas être une explication complète de qu'il y a de mal en lui. De l'autre côté, cependant, la seconde semble déjà contenir l'idée que l'acte est mal, et ne peut donc pas expliquer ce qu'il y a de mal en lui. Supposons, par contre, que nous options pour la conception absolutiste des droits, selon laquelle les spécifications adéquates de nos droits doivent contenir des qualifications telles qu'elles excluraient toute exception apparente à la portée de ces droits. Dans ce cas, la première difficulté refait surface : les mêmes croyances morales que nous essayons d'expliquer sont utilisées pour expliciter l'étendue du droit auquel nous faisons appel en tant qu'explication.

Alors, peu importe l'interprétation que nous adoptons concernant les droits, nous semblons en arriver au même problème : un explanans qui est luimême basé sur l'explanandum, et qui, donc, n'est pas véritablement capable de l'expliquer. Il faut préciser que l'objectif ici n'est pas d'argumenter pour une conception non-absolutiste des droits en opposition à une spécification plus nuancée des droits qui demeureraient encore absolus. La position que ce texte soutient est que peu importe l'option que nous prenons, un simple appel aux droits ne suffit pas pour expliquer nos engagements déontologiques. Dans l'une et l'autre de ces approches, nos jugements sur ce qui est admissible — opinions qui expriment les engagements déontologiques que nous cherchons à expliquer - jouent un rôle important dans l'explanans. De telles « explications » ne nous aideront pas à mieux comprendre notre conception morale.

\section{Ouvrages cités}

Dworkin, Ronald, 1977, Taking Rights Seriously, Cambridge, Mass., Harvard University Press.

Foot, Philippa, 1985, « Utilitarianism and the Virtues », Mind, 94, p. 196-209.

Scheffler, Samuel, 1988, "Introduction ", dans Scheffler, Samuel, dir., Consequentialism and Its Critics, Oxford, Oxford University Press.

Thomson, Judith Jarvis, 1976, "Self-Defense and Rights ", dans Thomson

1986a, publié la première fois sous Lindley Lecture, University of Kansas

Thomson, Judith Jarvis, 1986a, Rights, Restitution and Risk, Cambridge, Mass., Harvard University Press.

Thomson, Judith Jarvis, 1986b, "Afterword ", dans Thomson 1986a.

Thomson, Judith Jarvis, 1990, The Realm of Rights, Cambridge, Mass., Harvard University Press.

Thomson, Judith Jarvis, 1997, "The Right and the Good ", The Journal of Philosophy, XCIV, p. 273-298. 